\title{
Implementation of Low Cost Remote Primary Healthcare Services through Telemedicine: Bangladesh Perspectives
}

\author{
Uzzal Kumar Prodhan $^{1}$, Tushar Kanti Saha ${ }^{2}$ \\ Rubya Shaharin $^{3}$ \\ Department of Computer Science and Engineering \\ Jatiya Kabi Kazi Nazrul Islam University \\ Trishal, Mymensingh, Bangladesh
}

\begin{abstract}
In this paper, we have implemented a low cost primary healthcare service for the remote rural people of Bangladesh. These services were delivered through our developed advanced telemedicine model. The main aim of this paper is to provide basic healthcare service through the developed low cost hardware. We have developed Arduino based low cost hardware's to be used for this telemedicine services. Remote patients of Bangladesh can get the expert doctors opinion without going to the urban areas. We have collected nine vital signs such as electrocardiogram (ECG), oxygen saturation (SPO2), blood pressure, temperature, body position, glucose level, airflow, height, and weight of patients to be used in our model. We have removed unwanted signals from the collected vital signs through several filtering algorithms. Our system was successfully tested with the patients of Marie Stopes Bangladesh Hospital. From our developed model, rural patients can get primary healthcare services from the pharmacy of any remote village of Bangladesh with the assistance of local doctor by using Raspberry PI. Finally, we can say that the deployment of the developed healthcare service will reduce the cost of the telemedicine services and advances the healthcare facilities for the remote people of Bangladesh.
\end{abstract} SPO2

Keywords-Raspberry PI; DGHS; Arduino; Portable; ECG;

\section{INTRODUCTION}

Healthcare services of Bangladesh are mainly divided into two areas: urban and rural. Most of the expert doctors are urban centric where healthcare services are available. But, most of the people of Bangladesh live in rural areas. There are limited healthcare facilities in rural areas. There is a huge shortage of expert doctors in rural areas. As a result, remote rural people will have come to the urban areas for better healthcare services. Patients will have to travel to a long distance. They have to reside in city for some days to get treatment. The fees of expert doctor and different service costs will have to bear of patients. As a result, total costs are very high for the patients. Sometimes, it is also very difficult for the remote patients to go to the urban areas for the treatment due to the physical conditions of the patients. Fig. 1 shows the real scenario of health workforce-population situation of Bangladesh. From Fig. 1, we see that this ratio is very low for

\author{
Toufik Ahmed Emon ${ }^{4}$, Mohammad Zahidur Rahman ${ }^{5}$ \\ Department of Computer Science and Engineering \\ Jahangirnagar University \\ Savar, Dhaka 1212
}

Bangladesh. This ratio is also very low in the rural areas in comparison with the urban areas of Bangladesh.

Fig. 2 shows the comparative analysis of physician density among the western pacific regions. From Fig. 2, we can see that this ratio is very low for Bangladesh. Considering these scenario, telemedicine can be an alternate solution for the delivery of effective medical services to the large rural communities of Bangladesh.

Healthcare facilities are distributed in Bangladesh according to the Fig. 3. From Fig. 3, we can see that health services are distributed into five layers in Bangladesh. At present, telemedicine services are available up-to the selected hospitals in Upazilla level. So, there are huge scopes to introduce telemedicine services in Union and Ward level of Bangladesh.

\begin{tabular}{|c|c|c|}
\hline S.N. & Description of the facilities & Ratio \\
\hline \hline 1 & No. of doctors per 10,000 population & 1.43 \\
\hline 2 & No. of registered nurses per 10,000 population & 2.90 \\
\hline 3 & No. of registered physicians per 10,000 population & 4.90 \\
\hline 4 & No. of nurses per 10,000 population & 1.05 \\
\hline 5 & No. of medical technologists per 10,000 population & 0.37 \\
\hline 6 & $\begin{array}{c}\text { No. of community and domiciliary health workers per 10,000 } \\
\text { people }\end{array}$ & 4.04 \\
\hline 7 & Hospital bed for 1528 population & 1 \\
\hline
\end{tabular}

Fig. 1. Health Workforce-Population Status of Bangladesh.

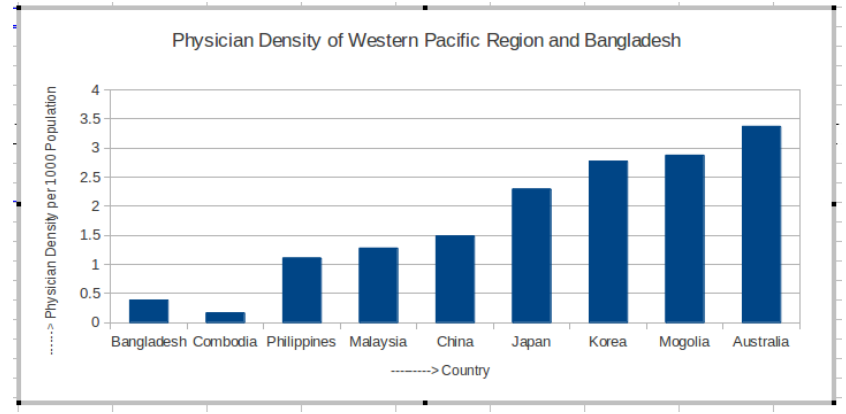

Fig. 2. Physician Density of the Western Pacific Countries. 


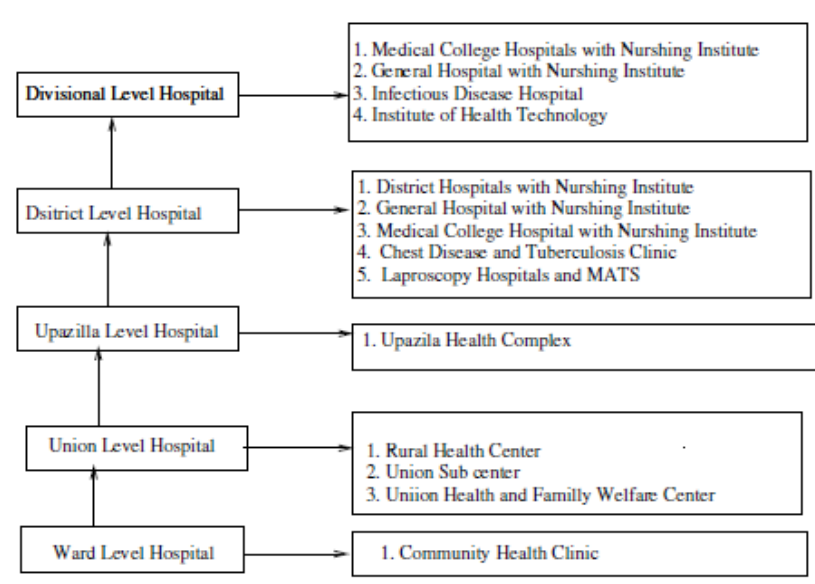

Fig. 3. Current Health Structure of Bangladesh.

Telemedicine can be an effective solution for addressing the current healthcare problems in Bangladesh. The scarcity of expert doctor in remote areas can be removed through telemedicine. Currently noninvasive technologies [1, 2-3] are combined with disease management process to provide more patient information, with a view to improve healthcare decision-making.

In this research, we have developed low cost remote primary healthcare services through telemedicine for addressing the financial problems of poor patients of Bangladesh. Remote people will go to the pharmacy of a remote village for their primary healthcare services. The pharmacy will be equipped with the developed necessary hardware's for telemedicine services. Local doctors will use our developed low cost portable telemedicine tool kit for the services. Patient's registration and telemedicine services will be delivered through the local pharmacy.

The rest of the paper is organized as follows: Section 2 is the review of literature section which presents the ongoing research activities done on the telemedicine and summarizes the scopes of further research, Section 3 is the materials and methods section which depicts the working methods of the proposed research and the corresponding materials, Section 4 is the results section which presents the outputs of this results, Section 5 is the discussion section which summarizes the research and Section 6 is the conclusion section which concludes the research.

\section{REVIEW OF LITERATURE}

This section presents some of the recently published papers with a view to find the current status of the research. Literature review helps us to find out the current demand of proposed research and presents the requirement analysis of the research. Now, we are going to discuss some of the recently published research in the section below.

A health system was proposed and developed by Ashir Ahmed and other researchers in 2013 through Portable Health Clinic and GramHealth. They have tested their system in three villages of Bangladesh and found satisfactory result for pilot project. In that paper they have tried to managed the huge amount of patient data to be used for fruitful health solution in a cost effective way [4]. Jecinta Kamau et al. developed a model "Social Services" on Wheel with a view to enhance the services in 2014 for the unreached people. This model [5] was tested in rural community of Bangladesh. The model was operated by subsidized but their proposed policies could make the model cost effective.

Scalable and Internet-based architecture for ECG in telemedicine application is developed by Umme Sayma Busra and others in 2014 to include hospitals and medical specialists to focus the rural health centers. In this research, the authors [6] used an ECG kit to measure the signal of a patient and send data to a server. The data is then forwarded to the nearest health professionals for measurement and provide consultation to the patients.

Aditi Kaushik and Pooja Sabherwal developed an ECG monitoring system by using android phone in 2015. They have used Pam Tompkins algorithmic approach. In this research work, feature extraction was done from the ECG signal. After feature extraction, its implementation was done in an android environment. Effective results are derived from this proposed research [7]. The research conducted by Salman Ahmed and others in 2015 results the construction of a device that can be used for transferring the data of a patient's vital signs to a remote device wirelessly. This research used blue-tooth technology for the transmission of data to expert doctors for evaluation. Temperature and ECG signal are taken from the patients to be analyzed and processing for transmitting. In future, authors [8] want to use GSM technology for data transmission to a wide range.

The authors presented a wearable sensor based elderly home care system in a smart environment for the elderly people in 2015. They have used PC as monitoring system, sensors for sensing data, web-service to communicate between monitoring systems. They have used two modules wearable user and main module for this research. They got satisfactory results for this model [9]. According to Amanda Mohon Ghosh and others remote patient monitoring and guidance awareness in a authenticated manner are the vital in telemedicine application. The whole work conducted by the researchers was carried out by three modules called sensing, main module and interaction module. This research [10] was evaluated in the hospital with the real patients for its acceptance.

ECG is one of the most important vital signs of noninvasive technique in diagnostics of heart oriented diseases [11]. Noise of ECG signal can be removed through Least Mean Square algorithm and hardware based filtering techniques [12]. Blood pressure (BP) is identified as a vital sign for controlling hypertension and cardiovascular disease [13]. Widely used blood pressure measuring technique is the oscillometric method through automated non-invasive BP measurement devices [14-15]. A study was conducted by Nilanjan Dey et al. [16] on residential wireless sensor networks for ECG healthcare monitoring in 2017.

Real time activity recognition framework for health monitoring support in home environments is developed by Shaikh Farhad Hossain in 2017. This system collects and analyzes sensory data in real-time. It gives variety feedback to the users. In addition, it can generate alerts based on the 
detected events and finally store the data to a server for further use [17]. Remote health monitoring system in a rural population: challenges and opportunities were conducted by Jacey-Lynn Minoi and Alvin W Yeo in 2014. In this study, blood pressure sensor is used for the pilot testing. Authors found that remote blood pressure monitoring is suitable for the management of hypertension in tele-homecare [18]. In 2016, Rafhanah Shazwani Binti Rosli et al. developed mobile heart rate detection system. This study develops a program for Arduino micro-controller that enables a pulse sensor module to detect alarming changes of a user's heart rate. They used a GM Shield to act as an SMS interface between the device and receivers. The developed is small sized and low cost which makes this device to be used more widely [19]. W. Yan [20] found that technology assists in meeting primary healthcare goals to understanding public healthcare difficulties, or to power individuals to engage in their own health. Authors [21] found the healthcare data breaches in this paper and they also found their primary causes with the role of PHI and HIPAA Act for dissuading data breaches. Authors [22] designed a healthcare system considering privacy and security through Blockchain technology for serving with deliberate medical care and benefits to the patients across. Authors [23] worked on the hiring of proper human resources and highly skilled professionals in the supply chain departments to improve the quality of supply chain healthcare services. Authors [24] proposed resource-aware service-oriented service model as a way to design systems that can efficiently provide quality healthcare services in this paper.

From the literature review section, we have found that real time vital information of remote patient is necessary for the expert doctor to deliver medical services. As we have limited doctors in rural areas, we are going to connect with the expert doctor in urban areas with the local doctors in a village with the low cost portable telemedicine tool kit. As a result, poor patient can enjoy the healthcare facilities at low cost from the remote pharmacy.

\section{MATERIALS AND METHODS}

In this section, we have developed the primary healthcare model to be deployed in the rural areas of Bangladesh. The model is shown in Fig. 4. The model is expressed through Business Process Modeling Notation (BPMN 2). There are four processes in our developed model for Bangladesh. The processes are local administrator in pharmacy, remote local doctor, expert doctor and health system administrator. The activities of the process are shown in Fig. 4. At first, we have developed the components of telemedicine model at low cost. According to the model, pharmacy is the local hub for the telemedicine services. Patients will come to the pharmacy of a local village for receiving telemedicine services. Administrator in pharmacy is responsible for inputting the patient's record to the health system. Local doctors in village are equipped with our developed portable tool kit. He is responsible for doing the primary health check-up of the patients and interacts with the expert doctor. Expert doctors will log-in to the health system through our developed client module. We have used Raspberry PI 3 Model B to make our remote client log-in module. All the medication plan and services will be given to the patients in a printed form from the pharmacy and the fees for the service is also received by pharmacy administrator.

The arrangements of the components of the developed telemedicine model for Bangladesh are shown in Fig. 5. From Fig. 5, we can see that there are six main components of the model. The components are sensors, developed portable telemedicine tool kit, android application, staging server, HL7 based health system, Raspberry PI based client log-in module. The sensor components are used to collect different vital signs of patients.

Portable telemedicine kit is developed with ardunio uno, ehealth sensor shield and Bluetooth. This kit is very small in size, low power consumption, easily portable and has easy interface with the sensors. This tool kit is powered from USB. As a result, we can easily use this kit in rural remote areas. Physical arrangement of the toolkit for Bangladesh is shown in Fig. 6. According to our design, this toolkit will be used by the local doctors for collecting vital signs of patients. In order to complete this task, we have used ardunio uno as the microcontroller.

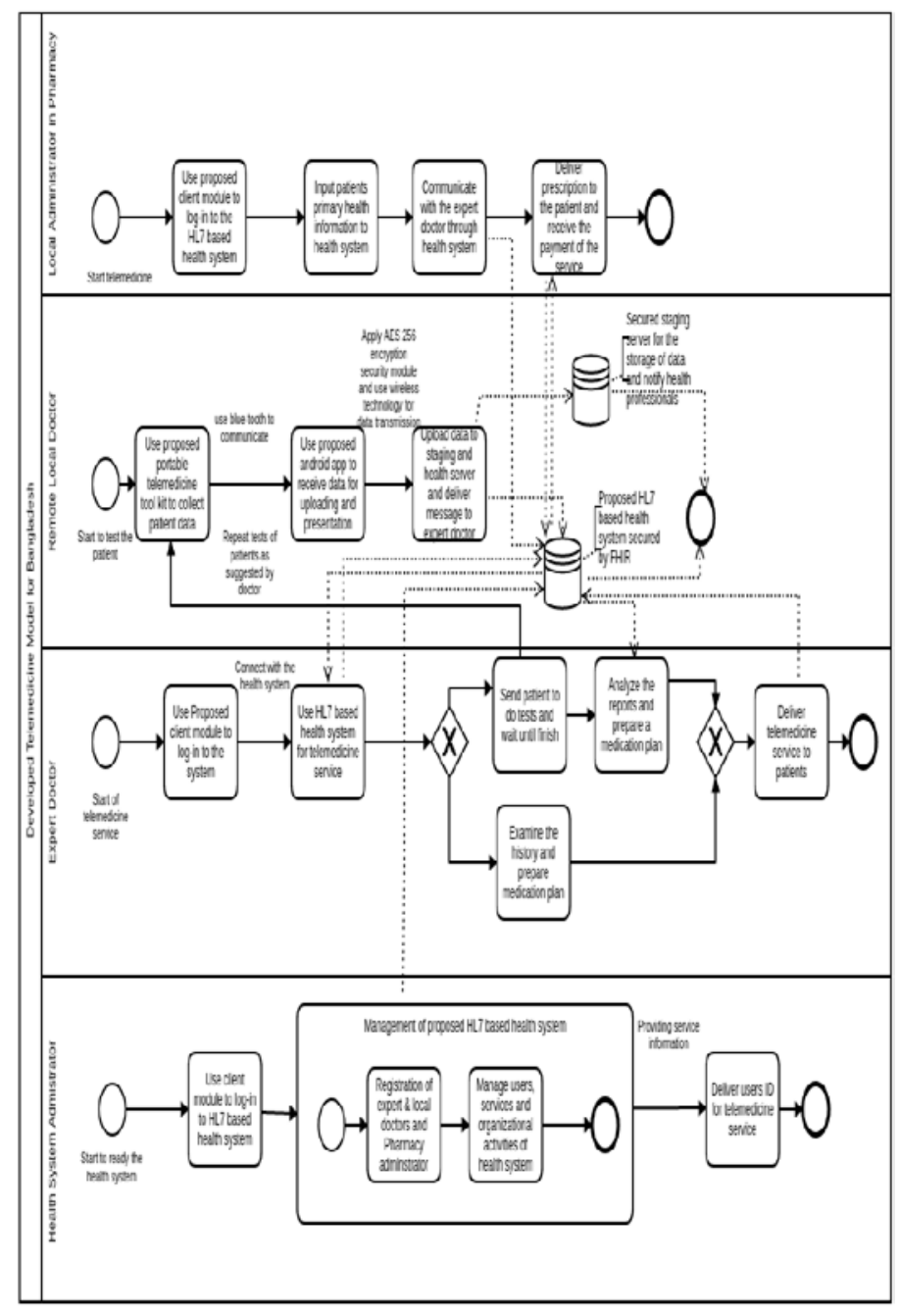

Fig. 4. Developed Primary Healthcare Model for Bangladesh. 
Sensors(ECG, SPO2, Airflow, Body Position, Glucometer, Blood Pressure, Temperature, Height, Weight)

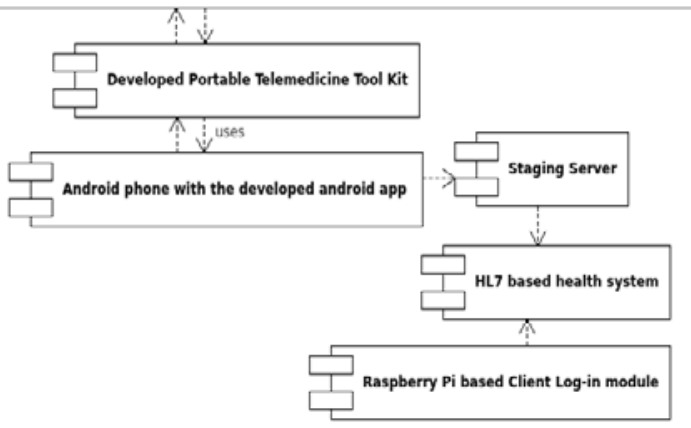

Fig. 5. Arrangements of the Components of Primary Healthcare Model.

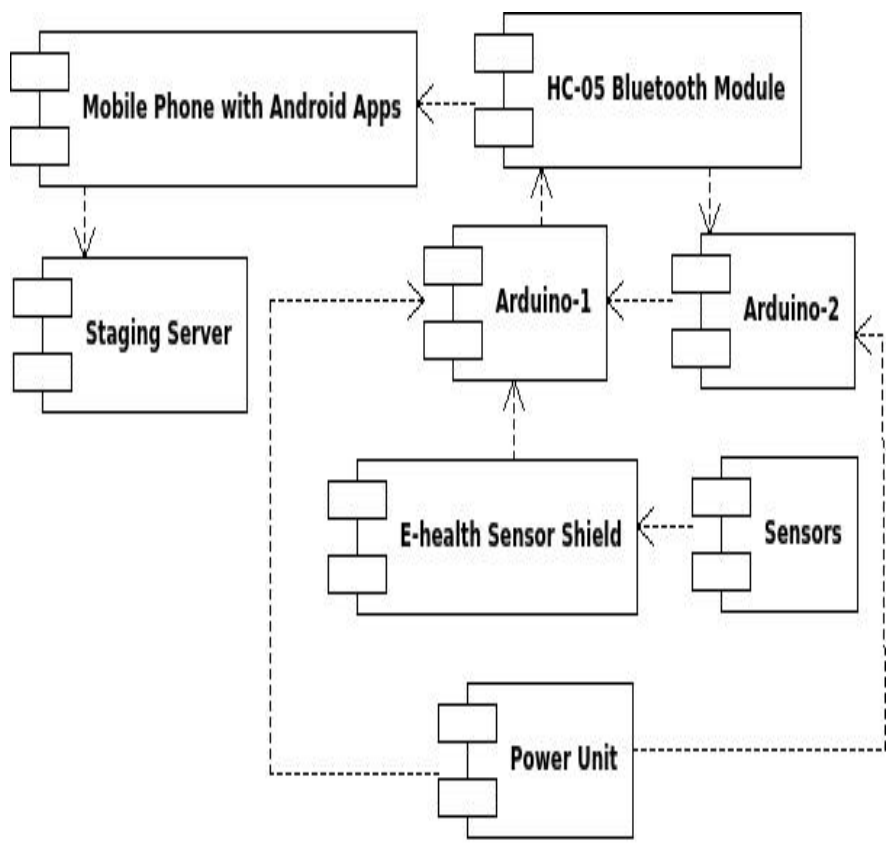

Fig. 6. Physical Arrangements of the Components of the Developed Toolkit.

We have developed a communication module for data processing, visualization and uploading. In order to do this work, we have developed an android application which will interact with our developed portable tool kit through Bluetooth. A secured valid connection is required to transfer patient data for processing and other works. We have done required preprocessing on the collected data to be used for telemedicine services. In this case, we have used AES encryption algorithm for preventing unauthorized access and securely data uploading to the staging server. Fig. 7 shows the user interface of our developed android application for our system.

In this research, we have used staging server for storing the field test data to be used by our health system, health professionals and other health organizations. Local doctors use portable kit and an android app to upload patient-wise lab test record to the server. In the server, we have used another messaging module for the expert doctor. Automated instant messages are delivered to expert doctors when any patients test data are available in the system. Quick remote healthcare services can be given to the patient by using our services. From the staging server, we have collected remote patient's vital information through python script. We have mapped the patient ID and test ID in both health system and staging server to import health record of patients.

From the present telemedicine models, we have seen that there are no health service related record is available in these models. As a result, the facilities of telemedicine services are not utilized fully. Patient health data are not interoperable among health organizations. This creates a lot of problems for us. In our model, we have used a HL7 based open source health system for telemedicine services. Our used health system is inter-operable and provides maximum benefits for the patients. All health service oriented electronic record is organized according to the definition of standard developing organizations. The modules of our used health system are shown in Fig. 8.

From the Fig. 8, we can see that there are mainly nine modules in our health system. Different health services are delivered remotely under these modules through our model. We have tried to reduce the overall cost of the system through the use of Raspberry PI. It is small in size, portable, easily manageable and a cost effective component for our model. We have made our remote log-in module for our developed system through Raspberry PI. Fig. 9 shows the developed remote client log-in module.

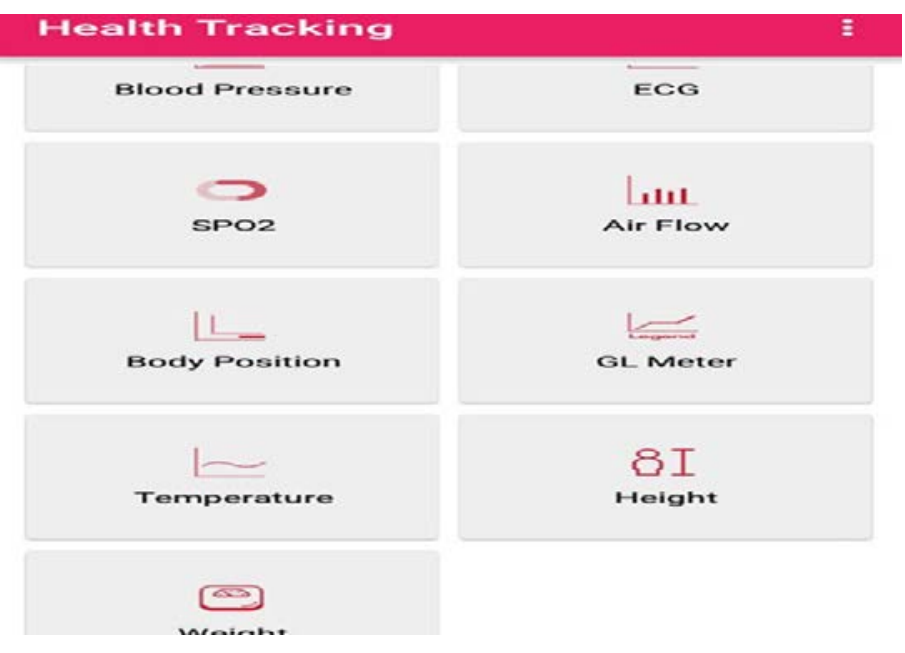

Fig. 7. User Interface of the Developed Android Application.

\begin{tabular}{l}
\hline \multicolumn{1}{c}{ Tryton-Administrator-Khidmah Hospital [Taka] } \\
\hline File User Options Favorites Help \\
\hline Search \\
\hline+8 Party \\
+ Product \\
+ Financial \\
+ \$E Currency \\
+ E Inventory \& Stock \\
+ Purchase \\
+ 3I Calendar \\
+ Health \\
+ A Administration
\end{tabular}

Fig. 8. Modules of Developed Health System. 


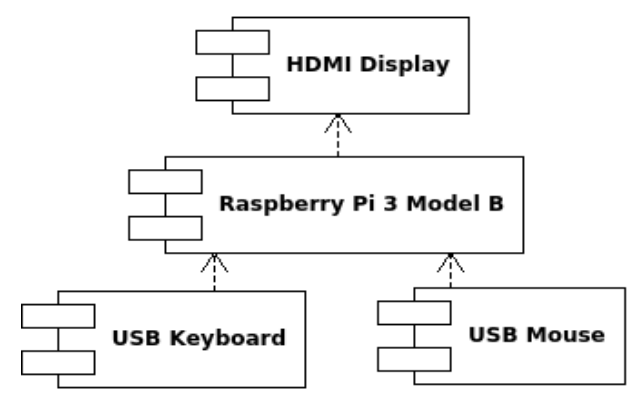

Fig. 9. Developed Raspberry PI based Client Module.

In order to make our model more secured, we have applied the suitable security features of standard and well known security model in our developed model. In our developed model, we have used the functionality of role based security model, multilevel based security model and access control list security model. Fig. 10 shows the security measurement techniques applied to our model. These techniques prevent unauthorized access and provide more privacy of the system.

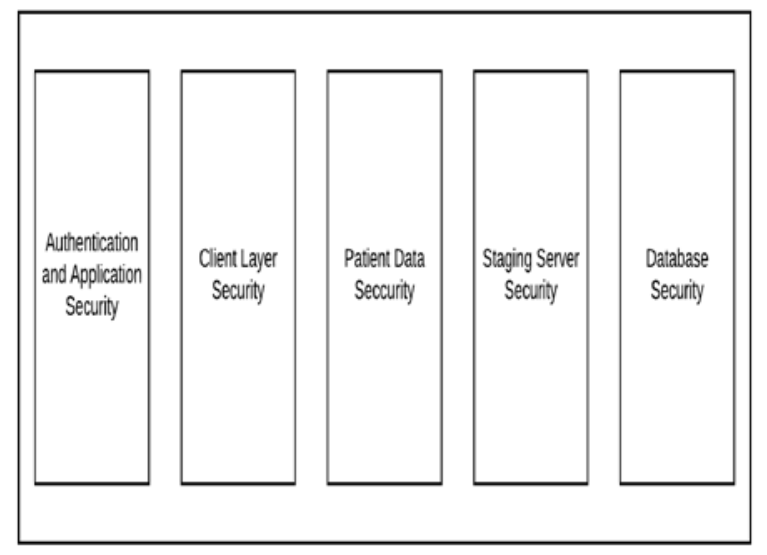

Fig. 10. Security Implementation Stages for the Developed System.

\section{RESULTS}

We have developed our components according to the telemedicine model shown in Fig. 4. We have installed the hardware components and successfully up-loaded the code to the microcontroller. We have prepared client module Raspberry Pi to connect with the health system. This log-in module will be used in the pharmacy of a local village. In this research, we have focused the primary healthcare services for the remote patients of Bangladesh. Our developed module works on blood pressure, ECG, height, weight, SPO2, airflow, body position, temperature, and glucometer sensor. Fig. 11 shows the log-in module for doctor to the health system.

Patient registration and history of the patient will be inputted by the administrator of pharmacy. Fig. 12 shows the snapshot from pharmacy module about patient record.

Remote patient will also get the medication plan from the expert doctor. Fig. 13 shows the medication report prepared by expert doctors and delivered from pharmacy module. Doctors can easily get the patients history and previous medication plan any time from the system. This can be a guide for them about the treatment of a patient.

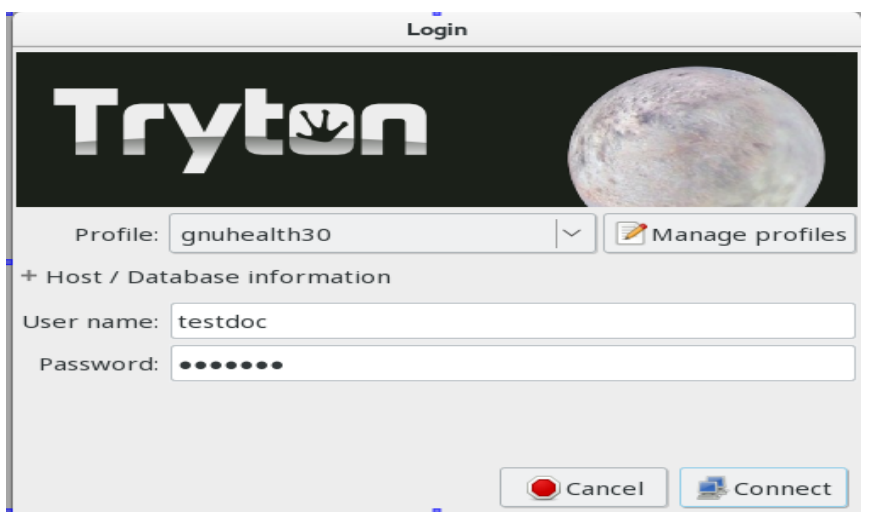

Fig. 11. Doctors Log-in Module of the System.

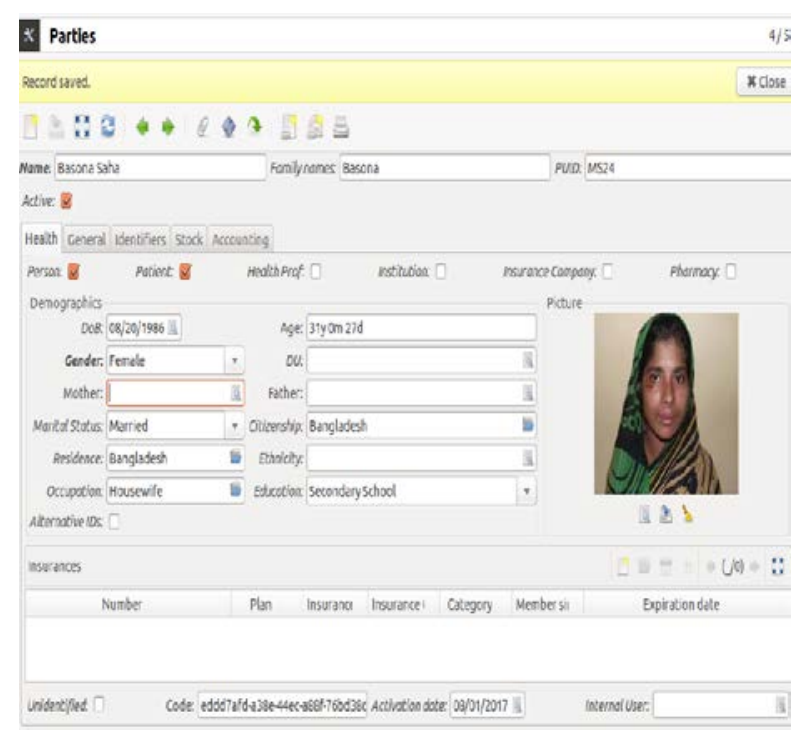

Fig. 12. Outputs from Pharmacy Module about Patient Record.

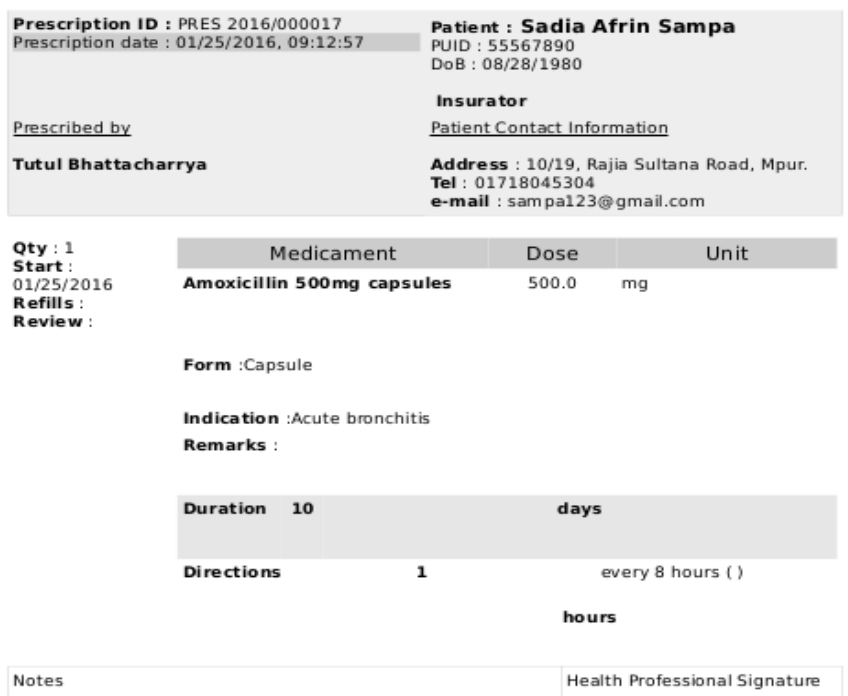

Fig. 13. Medication Report Generated from the Developed System.

Local doctor will use our developed android application to collect vital signs from the tool kit for further analysis. Fig. 14 
shows patient-wise data up-loading section to the staging server.

According to the direction of the expert doctor, local doctor will use our developed different sensors for data collection. Fig. 15 shows the output generated from our communication module for body position and glucometer sensor. Expert doctors will communicate with local doctors in case of emergencies for the better treatment of the patients. This communication will be done through our used health system.

Expert doctors are one of the vital actors in our model. When rural patient data are available through our communication module, the doctor is instantly notified through our messaging module. On line and off-line messaging are available in our system. Both e-mail and mobile number is used for the messaging of expert doctors. Doctors can easily log-in to the health system through our developed module. Doctors can instantly check the status of the patients and provide expert opinion to the remote patient. Fig. 16 shows the expert doctor's lab test requests for rural patients.

Warning messages can also be given in the report as well as real time message to expert doctor for the patients to take advance preparations. Fig. 17 shows the section of how the messages can be given to the patients and doctors for lab test results.

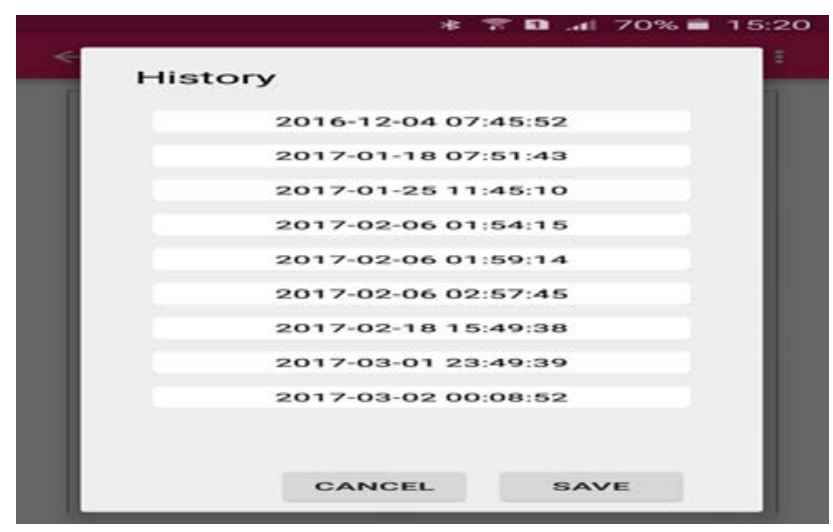

Fig. 14. Patient-wise History from Staging Server.

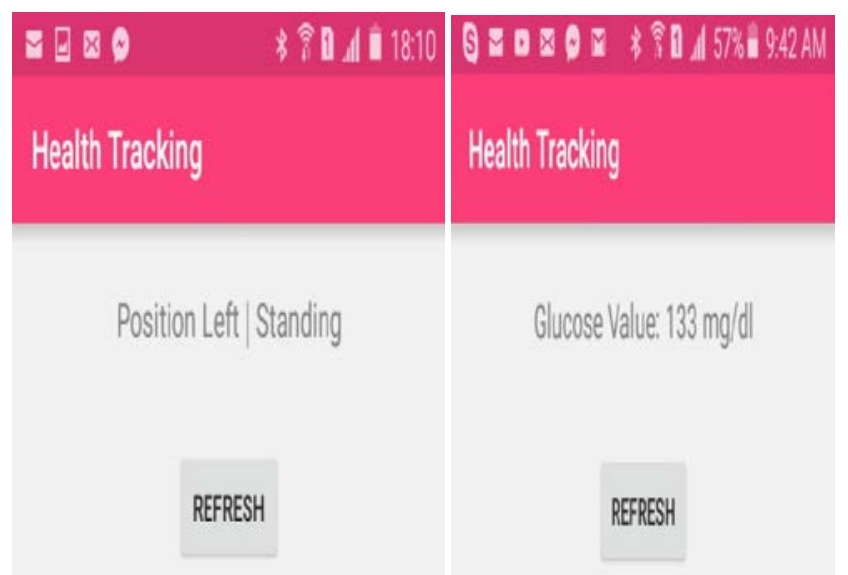

(a) Body Position Sensor Output. (b) Glucometer Sensor Output.

Fig. 15. Outputs from Body Position and Glucometer Sensor.
\& Lab Test Requests

1/51

\begin{tabular}{|c|c|c|c|c|c|c|}
\hline \multicolumn{6}{|l|}{ Fless Seard } & ty $100+$ \\
\hline Request & Testijpe & Dett & Dost & Patien: & Doccor & State \\
\hline & 49 Héght & $0,2 / 2 / 2017$ & 151212 & Succiliter & testocic & Dert \\
\hline & 48 Height & $08 / 23 / 2017$ & 1511:36 & Popy Siddoue & Wul Bhattadrya & Dret \\
\hline & 47 Weight & $0,2 / 23 / 2017$ & 151:200 & Siepa & Nulbistradya & Daft \\
\hline & 40 wècht & $003 / 23 / 2017$ & 150435 & Retin & Nulbhaltidinge & Dedt \\
\hline & 45 Aiffors & $00 / 1 / 2017$ & $1635: 2$ & Nodra alter & Itul Bhattrdya & Ordared \\
\hline & 4 Kiffon & $0 / 1 / 1 / 2017$ & 1437,97 & Pepry Sddove & NulBhatidedyo & Ordaed \\
\hline & 13 Neffow & $08 / 101 / 2017$ & 163726 & Unneselna & Wulbhattadya & Ordered \\
\hline & 42 Alfow & $0801 / 2017$ & 143655 & Recega bitun & nullohatcodinga & ordered \\
\hline & 415002 & $00 / 201 / 2017$ & 1429.17 & Naja, Khondoke Maga & Whul Bhattadnya & Ordered \\
\hline & 4) 5902 & $0 \% 1 / 2017$ & 142843 & Nara Mondoker $1 / 2 a$ & noul Bhathodina & orded \\
\hline
\end{tabular}

Fig. 16. Lab Test Requests for Patients from Expert Doctor.

Lab Tests Results

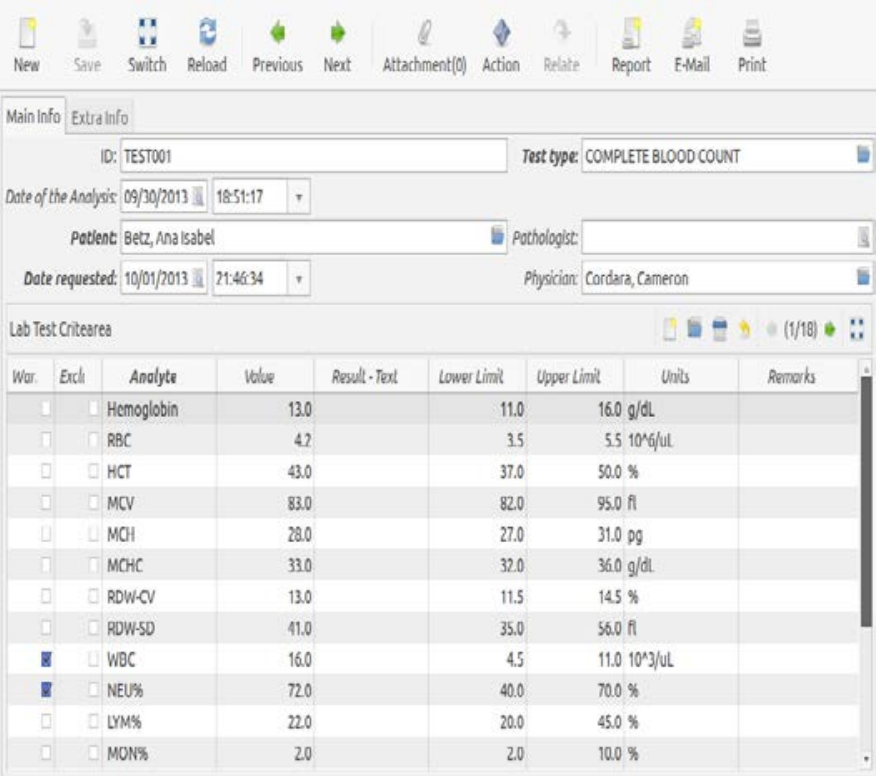

Fig. 17. Warning Message Generated from the System for the Patients.

\section{DISCUSSIONS}

In this paper, we have focused on the primary healthcare services for the huge number of remote rural people of Bangladesh. In order to meet their basic healthcare facilities, we have successfully developed the low cost components of the telemedicine service considering the financial status of these people. A recent study was done by Uzzal Kumar Prodhan and others in 2017 on the telemedicine services of Bangladesh. From the survey result, we came to know that the telemedicine operating cost should be in the range of 200-300 taka for the people of Bangladesh. Focusing on these financial proposals, we have developed a cost effective model so that remote people can afford the cost and our telemedicine model will be sustainable in-terms of cost.

Most of the telemedicine systems were in project basis in Bangladesh. Some of the models use real time based facilities such as video conferencing and some of the models use store and forward based techniques. But in our developed advanced model, we have included the facilities of both methods for the 
acceptance of our system. In the case of local doctors, they are getting the rural patient information through our communication module in real time. Then local doctors send the vital information to expert doctor through our health system by using store and forward based method. Expert doctors also provide the medication plan through store and forward based. By using this approach, we can deliver fast healthcare services to the remote patients.

From the survey results published in 2016, we came to know that rural patient costs minimum 1500 taka to get one time treatment from the expert doctors from urban areas. Sometimes it is also difficult or impossible for a remote patient to travel to a long distance. In order to assist those patients, we have developed this model which can be easily deployable in the pharmacy of a remote village. Patients can get the expert doctors opinion without travelling to urban areas from pharmacy. This arrangements saves rural patients time, cost and get immediate primary healthcare services at low cost.

Telemedicine service center setup cost is very high to introduce telemedicine services. In Bangladesh, these costs vary from project to project. Largest telemedicine projects run by Director General of Health Services (DGHS), Bangladesh costs about 30 lacks taka to setup a center. All the models were in hospital based. Patients will have come to the urban telemedicine center to get the service. We want to deploy our advanced telemedicine model to the remote areas of Bangladesh. So, we have considered their financial strength. Normally, a pharmacy needs to use a Raspberry Pi based client module and an Internet connection to run the center. This module will be connected to the centralized health system. This module costs around 10000 taka only. Pharmacy owner's has already showed their interest to invest this small amount for the health services of the poor people of Bangladesh.

In this research, local doctors are equipped with our developed tool kit for doing our different primary tests according to the direction of the expert doctor. We have focused on the primary health check-up of rural patients. By using our health kit, we can collect nine vital signs of patients and check the patient's primary vital signs in real time. We have included a HC-05 Bluetooth module to make our kit for sending data for further diagnosis. In this kit, we have used low cost microcontroller named ardunio uno and e-health sensor shield. The cost of the kit is also very low compared to the available existing tool kit used in our country. This tool kit has extensibility capabilities. We can add more sensors with our developed tool kit by updating the microcontroller code to diagnosis more vital parameters of patients.

In order to test our developed tool kit, we have conducted a field test with the patients of Marie Stopes Bangladesh Hospital, Dhaka, Bangladesh. Our sample size was twenty. We have tested our system with the close supervision of the expert doctors in the hospital. Doctors have checked the collected results from our device and verified the results. The results are shown in the result section.

This research worked on nine vital signs of patients only. In future we can easily extend this research work to collect more vital signs of patients. This study conducted only one field test with the Marie Stopes hospital. More field test can be conducted for the validity of the developed system.

Every health organizations in Bangladesh have its own customized health systems. Currently, health systems of Bangladesh do not interact with each other for health services. As a result, patients will have to cost more for the disparity of present health systems. They will have to do their same health check-up repeatedly. There is no central database of their health record. All these lacking make the total health system inefficient. In this study, we have solved these problems by following the recommendations of standard developing organization for health. In order to offer telemedicine services, we have followed Health Level Seven International (HL7) based open source health system. This health system solves data interoperability problem and assists health professionals to make a decision about a patient. This approach helps health organizations to manage their patient's records efficiently.

\section{CONCLUSIONS}

We have successfully implemented and tested our developed telemedicine model for primary healthcare services of remote patients. In this research, we also developed the required hardware's at low cost. We have used Bluetooth and mobile network for collecting rural patient data to be used in our advanced telemedicine model. As mobile network is available in all the remote villages of Bangladesh, we have used these features in remote client module for telemedicine services. Remote client module can be operated with low energy. As power crises exist in Bangladesh, we can run this module without electricity for a long time. Pharmacy will be the service center for this model where patients will receive their services. Local doctors, patients and expert doctors are the vital components of the advanced telemedicine model. Remote patients can get the improved healthcare services at low cost without travelling to a long distance. Expert opinion can be easily and instantly delivered to the patients in emergency situation. Finally from the point of view of Bangladesh, we can say that this model will be a cost effective and sustainable telemedicine model for the telemedicine services for the unreached people of Bangladesh.

\section{ACKNOWLEDGMENT}

Authors would like to thanks to the Information and Communication Technology Division of Ministry of Posts, Telecommunications and Information Technology, ICT Tower, Agargoan, Dhaka, Bangladesh for their fellowship of $\mathrm{PhD}$ program. Authors would also like to give especial thanks to the officials of Computer and Electronics labs of Jahangirnagar University, Savar, Dhaka, Bangladesh for their assistance to make this research successful.

\section{REFERENCES}

[1] Caroline Free, Gemma Phillips, Louise Watson, Leandro Galli, Lambert Felix, Phil Edwards, Vikram Patel, and Andy Haines, “The effectiveness of mobile-health technologies to improve health care service delivery processes: A systematic review and meta-analysis,” PLOS, Vol. 10, No. 1, 2013, e1001363.

[2] Steinhubl SR, Muse ED, and Topol EJ, "Can mobile health technologies transform health care?,” JAMA, Vol. 310, No. 22, 2013, pp. 2395-2396. 
[3] Steven R. Steinhubl, Evan D Muse, and Eric J. Topol, "The emerging field of mobile health," Science Translational Medicine, 7(283):283rv283, 2015.

[4] Ashir Ahmed, Lutfe Kabir, Eiko Kai, and Sozo Inoue, "Gramhealth: A bottom-up approach to provide preventive healthcare services for unreached community,” in Annual International Conference of the IEEE EMBS, No. 35, IEEE, 2013, pp. 1668-1671.

[5] Jecinta Kamau, Andrew Reberio-Hargrave, Hiroaki Satto, Emran Abdullah, Hiroshi Okajima, and Ashir Ahmed, "Social services on wheels: A sustainable model to improve access in unreached communities," in IST-Africa 2014 Conference, 2014, pp. 1-8.

[6] Umme Sayma Busra and Mohammad Zahidur Rahman, "Mobile phone based telemedicine service for rural Bangladesh:ECG," in International Conference on Computer and Information Technology, No. 16, IEEE, 2014, pp. 203-208.

[7] Aditi Kaushik and Pooja Sabherwal, "Monitoring electrocardiogram using android based smart phone," in Annual IEEE India Conference, IEEE, 2015, pp. 1-6.

[8] Salman Ahmed, Sabrin Millatand, Md. Aymanur Rahman, Sayeda Naeyna Alam, and Md. Saniat Rahman Zishan, "Wireless health monitoring system for patients," in IEEE International WIE Conference on Electrical and Computer Engineering, IEEE, 2015, pp. 164-167.

[9] Md.Nazam Al Hossain, Aprojit Pal, and SK Alamgir Hossain, “A wearable sensor based elderly home care system in a smart environment," in International Conference on Computer and Information Technology, No. 18, IEEE, 2015.

[10] Ananda Mohon Ghosh, Debashish Halder, and SK Alamgir Hossain, "Remote health monitoring system through IoT," in International Conference on Informatics, Electronics and Vision, No. 5, IEEE, 2016, pp. 921-926.

[11] V.Vijendra and Meghana Kulkarni, "ECG signal filtering using dwt haar wavelets coefficient techniques," in International Conference on Emerging Trends in Engineering, Technology and Science, IEEE, 2016, pp 1-6.

[12] Laxmi Shetty, "Electrocardiogram preprocessing using Weiner filter and least mean square algorithm," International Journal of Innovative Research in Electrical, Electronics, Instrumentation and Control Engineering, Vol. 3, No.1, 2015, pp. 82-85.

[13] Wilmer W Nichols, Charalambos Vlachopoulos and Michael O Rourke, "Mc-Donald Blood Flow in Arteries, Sixth Edition: Theoretical, Experimental and Clinical Principles," CRC Press, Editions 6, 2011.

[14] M L Antonova, "Noninvasive determination of the arterial elastogram and blood pressure part i: Arterial elastogram and volume pulsations," Blood Pressure Monitoring, Vol. 18, 2013, pp. 32-40.
[15] José Antonio de la O Serna, Wendy Van Moer, and Kurt Barbé, “Using alternating kalman filtering to analyze oscillometric blood pressure waveforms," in IEEE Transaction Instrument Measurement, Vol. 62, IEEE, 2013, pp. 2621-2628.

[16] N. Dey, A. S. Ashour, F. Shi, S. J. Fong, and R. S. Sherratt, "Developing Residential Wireless Sensor Networks for ECG Healthcare Monitoring," in IEEE Transactions on Consumer Electronics, Vol. 64, No. 4, 2017, pp. 442-449.

[17] Shaikh Farhad Hossain, "Real time activity recognition framework for health monitoring support in home environments," in 3rd International Conference on Electrical Information and Communication Technology (EICT), Khulna, IEEE, 2017, pp. 1-6.

[18] J. L. Minoi and A. W. Yeo, "Remote Health Monitoring System in a Rural Population: Challenges and Opportunities,” in IEEE Conference on Biomedical Engineering and Sciences, 2014, pp. 895-900.

[19] R. S. B. Rosli and R. F. Olanrewaju, "Mobile Heart Rate Detection System for EarlyWarning of Potentially-Fatal Heart Diseases,” in International Conference on Computer and Communication Engineering, IEEE, 2016, pp. 422-427.

[20] W. Yan, "Technologies for Primary Health Care Help Meet Global Goals," in IEEE Pulse, Vol. 10, No. 3, pp. 15-18, May-June 2019, doi: 10.1109/MPULS.2019.2911822.

[21] A. Jayanthilladevi, K. Sangeetha and E. Balamurugan, "Healthcare Biometrics Security and Regulations: Biometrics Data Security and Regulations Governing PHI and HIPAA Act for Patient Privacy," 2020 International Conference on Emerging Smart Computing and Informatics (ESCI), Pune, India, 2020, pp. 244247,doi:10.1109/ESCI48226.2020.9167635.

[22] S. Chakraborty, S. Aich and H. Kim, "A Secure Healthcare System Design Framework using Blockchain Technology," 2019 21st International Conference on Advanced Communication Technology (ICACT), PyeongChang Kwangwoon_Do, Korea (South), 2019, pp. 260-264, doi: 10.23919/ICACT.2019.8701983.

[23] G. K. Getele, T. Li and J. T. Arrive, "The Role of Supply Chain Management in Healthcare Service Quality," in IEEE Engineering Management Review, vol. 48, no. 1, pp. 145-155, 1 Firstquarter,march 2020, doi: 10.1109/EMR.2020.2968429.

[24] K. Wan and V. Alagar, "Resource-aware Service-oriented Approach for Elderly Healthcare," 2018 5th International Conference on Systems and Informatics (ICSAI), Nanjing, 2018, pp. 1199-1205, doi: 10.1109/ICSAI.2018.8599300. 